\title{
Association of NTCP Gene Polymorphisms and Spontaneous Clearance of Hepatitis B Virus in Asia: A Meta-Analysis
}

\author{
Peipei $\mathrm{Hu}^{1}$, Jundi Liu ${ }^{1}$ and Dingmei Zhang ${ }^{1, *}$ \\ ${ }^{1}$ Department of Medical Statistics and Epidemiology, School of Public Health, Sun Yat-sen University, Guangzhou, China \\ "Corresponding author: Department of Medical Statistics and Epidemiology, School of Public Health, Sun Yat-sen University, Guangzhou, China. Tel: +86-2087330595, Email: \\ zhdingm@mail.sysu.edu.cn
}

Received 2019 May 27; Revised 2019 September 12; Accepted 2019 September 21.

\begin{abstract}
Context: It is reported that NTCP(sodium taurocholate cotransporting polypeptide) gene polymorphisms are associated with spontaneous clearance of hepatitis B virus (HBV), but the results are contradictory. Therefore, the current meta-analysis aimed at systematically clarifying the association between NTCP gene polymorphisms and spontaneous clearance of HBV in Asia.

Evidence Acquisition: PubMed, Embase, Web of Science, Scopus, China National Knowledge Infrastructure and China Wanfang Data were searched systematically for articles published before 09 August 2019.

Results: A total of nine case-control studies were finally included in the current meta-analysis. The pooled results indicated that the NTCP gene rs2296651 A allele is a protective factor for spontaneous clearance of HBV (GG vs. GA+AA: odds ratio (OR) $=0.73,95 \%$ confidence interval $(\mathrm{CI})=0.58-0.93 ; \mathrm{GG}$ vs. $\mathrm{GA}: \mathrm{OR}=0.74,95 \% \mathrm{CI}=0.59-0.93 ; \mathrm{G}$ vs. A allele: $\mathrm{OR}=0.74,95 \% \mathrm{CI}=0.58-0.94)$.

Conclusions: NTCP gene rs2296651 polymorphism is correlated with spontaneous clearance of HBV, while NTCP gene rs4646287 and rs7154439 variants are not associated with persistent HBV susceptibility.
\end{abstract}

Keywords: Hepatitis B Virus, Single Nucleotide Polymorphism, Sodium-Bile Acid Cotransporter

\section{Context}

Hepatitis B virus (HBV) infection is one of the major public health concerns worldwide, with a total of 248 million chronically infected patients (1); it is the main cause of cirrhosis and hepatocellular carcinoma. The outcome of $\mathrm{HBV}$ infection is varied: spontaneous clearance, HBV carriers, patients with chronic HBV infection, cirrhosis, or hepatocellular carcinoma, which is influenced by environmental, viral and host genetic factors. HBV infection exhibits a significant family clustering, and relatives with blood relationship are more susceptible to HBV infection than ones without blood relationship (2). In addition, studies by Lin et al. (3) showed the coincidence rate of identical twin HBV infection is higher than that of fraternal twins, which may mean that genetic factors were the important causes of HBV infection.

In recent years, the discovery of sodium taurocholate cotransporting polypeptide (NTCP) was a major breakthrough in the researches of HBV infection mechanism. NTCP is the coding product of SLC10A1, a member of the 10th solute carrier gene family. Early studies suggested that the main function of NTCP is to absorb and transported back to the stem cells at a 2:1 ratio of $\mathrm{Na}^{+}$reabsorbed by the portal vein to conjugated bile acid, which plays an important role in the intestinal-hepatic circulation of bile acid (4). Recent studies found that NTCP is a functional receptor of hepatocytes infected with HBV, which mediates HBV invasion and infection through specific binding with the pre-S1 antigen of HBV envelope protein (5). The study by Yan et al. (6) revealed that mutation of p.S267F (rs2296651) in SLC10A1 gene can decrease the expression level of NTCP or the affinity between NTCP and HBV; thereby, reducing the infection of HBV to hepatocytes. To date, a series of studies show a correlation between NTCP gene variants and HBV infection, but the results of some studies are contradictory. For instance, the study by Su et al. (7) focusing on Tibetans and Uygurs from West of China showed no association between SLC10A1 (rs2296651) polymorphism and HBV chronicity. In contrast, the investigation by Peng et al. (8) indicated that gene variant of NTCP was significantly correlated with resistance to chronic HBV infection.

\section{Objectives}

The current study mainly aimed at evaluating the association between NTCP gene polymorphisms and sponta- 
neous clearance of HBV in Asian population by summarizing the results of previous studies.

\section{Data Sources}

Two independent investigators systematically searched for articles studying the association between NTCP gene polymorphisms and HBV in the following databases: PubMed, Embase, Web of Science, Scopus, Chinese National Knowledge Infrastructure, and Chinese WanFang Data. The latest search update was 09 August 2019. The search strategy was relied on a combination of "NTCP OR sodium taurocholate cotransporting polypeptide OR SLC1OA1", "hepatitis B virus OR HBV OR CHB” and "polymorphism OR variant OR SNP OR gene mutation OR genetic variants", without any limitation of language or publishing year. In addition, the reference list of eligible articles and relevant reviews were manually searched to find other potential sources of studies.

\section{Study Selection}

\subsection{Inclusion Criteria}

Each study was independently assessed by two investigators, and the third party opinions were sought in case of disagreements. The main inclusion criteria in the current meta-analysis were: (1) evaluation of the association between NTCP gene polymorphisms and spontaneous clearance of $\mathrm{HBV}$; (2) reporting the number of genotypes in case and control groups, and providing sufficient information to calculate odds ratio (OR) and 95\% confidence interval (CI);(3) selection of HBV persistent infection as the case and spontaneous clearance of infection as the control groups, while the diagnostic criteria were clearly described; and (4) case-control study. The exclusion criteria were: (1) overlapping data, and (2) enrolling patients co-infected with HCV, HDV, or HIV. If there were multiple literature reports on the same population, only the largest study was included in the meta-analysis. The quality of literature was assessed by the Newcastle-Ottawa scale (NOS), which consists of three parts, as the selection of subjects ( 0 - 4 points), the comparability between groups ( 0 - 2 points), and the measurement of exposure ( 0 - 3 points) (Supplementary File Appendix 1).

\subsection{Definitions}

HBV persistent infection is defined as patients with positive HBsAg for more than six months. HBV spontaneous clearance refers to the ones negative HBsAg, but positive for HBsAb, without a history of vaccination and hepatitis.

\section{Data Extraction}

For each article included in the analysis, the following data were extracted: the first author, year of publication, country and ethnicity of participants, sample size, age, gender, allele and genotype frequencies of SNP, and genotyping methods. The two researchers extracted data independently and agreed on inconsistent contents through discussions with the third party.

\subsection{Statistical Analysis}

In the current meta-analysis, pooled OR and 95\%CI were performed for dominant (GG vs. GA+AA), recessive (GG+GA vs. AA), codominant (GG vs. AA, GA vs. AA, GG vs. GA) and alleles ( $G$ vs. A allele) genetic model to analyze the association between NTCP gene polymorphisms and spontaneous clearance of HBV infection. Since the number of AA genotypes of rs2296651 in some studies was zero in the case and control groups, the association of rs2296651 was only analyzed in dominant, codominant, and alleles models. First, Q-test and $\mathrm{I}^{2}$ statistic were used to assess heterogeneity between studies $\left(\mathrm{P}<0.1\right.$ and $\mathrm{I}^{2}>50 \%$ indicating significant heterogeneity). Subsequently, based on the results of the heterogeneity, the pooled OR value and the 95\% CI were calculated using the random effects model or the fixed effect model. The subgroup analysis was performed based on Hardy-Weinberg equilibrium (HWE) when significant heterogeneity was found or the frequency distribution of the control group gene in the study did not satisfy the HWE ( $P$ $<0.1)$. Next, the sensitivity analysis was used as a method of recalculating the combined OR values after omitting the studies one by one. Publication bias was measured by the funnel plots and Egger regression test. All of the above statistical analysis methods were performed by Stata V. 15.0 software (StataCorp, College Station, TX, USA). Unless otherwise stated, a two-tailed $\mathrm{P}<0.05$ was considered the level of significance.

\section{Results}

\subsection{Literature Retrieval and Literature Quality Evaluation}

A total of 201 literatures were retrieved. According to inclusion-exclusion criteria, nine case-control studies (715) published from 2014 to 2019 were finally included in the meta-analysis. The literature selection chart is shown in Figure 1. The meta-analysis included 14165 subjects, including 6400 spontaneous clearance controls and 7765 patients with persistent HBV infection (Supplementary File Appendix 2). In the association study of rs2296651 by Zhang et al. (13) $(\mathrm{P}=0.004)$ and Cheng et al. (14) $(\mathrm{P}=0.060)$, the frequency distribution of the control group gene did not satisfy the HWE, neither did the studies of Wu $(11)(\mathrm{P}<0.001)$ 
and Su et al. $(7,9)(P=0.024, P=0.035)$ in the rs7154439. The basic characteristics and quality scores of the included studies are shown in Table 1.

6.2. Association Between the rs2296651 Polymorphism and Spontaneous Clearance of $\mathrm{HBV}$

Considering the heterogeneity, the association between rs2296651 polymorphism and self-limited clearance of HBV was analyzed using the random effects model. Pooled results indicated that rs2296651 A allele was a protection factor against persistent $\mathrm{HBV}$ infection ( $\mathrm{G}$ vs. A allele: $\mathrm{OR}=0.74,95 \% \mathrm{CI}=0.58-0.94)$. The results were similar under the dominant model (GG vs. GA+AA: OR $=0.73,95 \% \mathrm{CI}$ $=0.58-0.93$ ) and codominant model (GG vs. GA: OR=0.74, $95 \%$ CI $=0.59-0.93$ ) (Table 2 ).

Next, subgroup analysis was performed according to whether the control population met HWE. In the allele model, the analysis results of both subgroups suggested the protective effect of A allele (met HWE: $\mathrm{OR}=0.62,95 \% \mathrm{CI}$ $=0.39$ - 0.97; out of HWE: $\mathrm{OR}=0.86,95 \% \mathrm{CI}=0.75-0.98)$. Yet, only the subgroups of the studies satisfying HWE showed significant association in the dominant model (GG vs. $\mathrm{GA}+\mathrm{AA}: \mathrm{OR}=0.60,95 \% \mathrm{CI}=0.40-0.91)$ and codominant model (GG vs. GA: OR $=0.61,95 \% \mathrm{CI}=0.43-0.85$ ). No significant heterogeneity existed among studies that were consistent with HWE under the codominant model. In the dominant model, after excluding studies by Zhang et al. (13) and Cheng et al. (14) due to deviation from HWE, the heterogeneity among the studies decreased ( $\mathrm{I}^{2}$ decreased from $51.1 \%$ to $46.3 \%$ ) (Figure 2 ).

\subsection{Association Between the rs4646287 Polymorphism and Spontaneous Clearance of $\mathrm{HBV}$}

Under all six genetic models, there was no significant heterogeneity among the studies. Thus, the fixed effect model was used for analysis. Pooled results indicated no association between rs4646287 polymorphism and spontaneous clearance of HBV infection (Table 2).

6.4. Association Between the rs7154439 Polymorphism and Spontaneous Clearance of $\mathrm{HBV}$

The correlation between rs7154439 polymorphism and self-limited clearance of $\mathrm{HBV}$ was analyzed by the fixed effects model (GG vs. GA+AA, GG vs. GA, G vs. A allele) and the random-effect model (GG+GA vs. AA, GG vs. AA, GA vs. AA) according to the degree of inter-study heterogeneity. No evidence indicated the association between rs7154439 polymorphism and self-limited clearance of HBV under all six genetic models (Table 2 ).

Subgroup analysis was conducted by recessive model and codominant model 1 and 2 according to whether the
HWE was satisfied. In the subgroups of the studies satisfying HWE, there was also no significant association between rs7154439 polymorphism and self-limited clearance of HBV. Results showed no significant heterogeneity within each subgroup (Figure 3).

\subsection{Sensitivity Analysis}

Sensitivity analysis was performed to assess the impact of each study on the pooled results by omitting each study separately in all genetic models. The results of the analysis of SNP of rs2296651 and rs4646287 indicated that no study had a significant impact on overall results. However, as for recessive model, codominant models 1 and 2 in rs7154439, after excluding studies of Zhang et al. (13), the results were reversed and showed the protective effect of $A$ allele against chronic HBV infection.

\subsection{Publication Bias}

The potential publication bias was evaluated by funnel plots and Egger regression test. The shape of all funnel plots did not reveal obvious asymmetry and Egger regression also indicated that there is no evidence of potential publication bias in all genetic models (Table 2, Figure 4).

\section{Conclusions}

A study by Yan et al. (6) found that the NTCP molecule with the S267F (rs2296651) mutation loses its ability to transport bile acids and bind to HBV-PreS1 and HBV. Yet, the effects of this mutation on HBV infection and subsequent liver disease in patients with chronic HBV are not fully understood. According to the results of the current meta-analysis, SLC10A1 rs2296651 A allele was a protective factor against HBV persistent infection in Asian populations. The frequency of genotype GA and AA in patients with persistent $\mathrm{HBV}$ infection were higher than those of the spontaneous recovery group, and the difference was statistically significant. Subgroup analysis and sensitivity analysis showed similar results. Furthermore, after omitting the study by Zhang et al. (13) and Cheng et al. (14) due to deviation from HWE, the heterogeneity among the studies decreased under the dominant and codominant model, indicating that the conclusion was robust and deviation from HWE may be one of the source of heterogeneity in the overall analysis. Interestingly, the current mutation of the rs2296651 was only observed in Asian populations with significant racial specificity. For instance, the S267F variant was absent among Moroccans both in healthy controls and patients with chronic HBV patients (16). Perhaps this is one of the reasons for the species-specificity of HBV infection. 


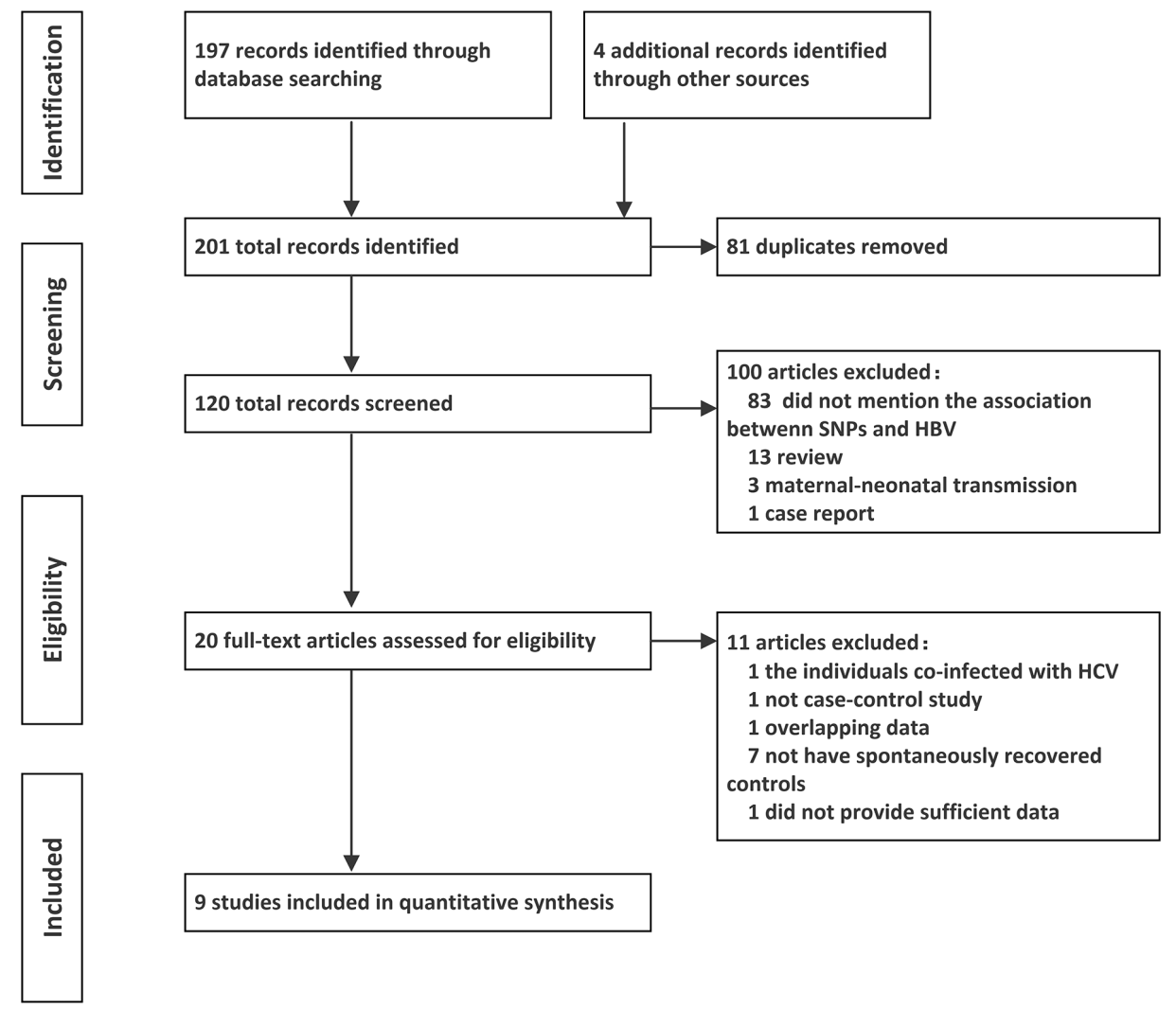

Figure 1. Flow chart of literature search and screening

\begin{tabular}{|c|c|c|c|c|c|c|c|c|c|}
\hline Study ID & Country & Ethnicity & SNP & Control & Case & $\begin{array}{l}\text { Gender, Male (\%, } \\
\text { Control/Case) }\end{array}$ & Age (Control/Case) & Method & NOS \\
\hline Su, Z. (2014)(9) & $\begin{array}{l}\text { Southwestern } \\
\text { China }\end{array}$ & Han & $\begin{array}{l}\text { rs4646287 } \\
\text { rs7154439 }\end{array}$ & 352 & 581 & $54.8 / 70.4$ & $(50 \pm 12)(47 \pm 13)^{\mathrm{a}}$ & TD PCR & 6 \\
\hline Peng, L. (2015)(8) & Southern China & Han & rs2296651 & 90 & 99 & $\mathrm{NA}$ & $\mathrm{NA}$ & Sanger & 7 \\
\hline Chen, $X .(2016)(10)$ & Central China & Han & rs7154439 & 713 & 1023 & $60.1 / 64.1$ & $37.3[2-82] / 36.2[3-81]^{\mathrm{b}}$ & TaqMan & 7 \\
\hline \multirow{2}{*}{ Su, Z. (2016) (7) } & \multirow{2}{*}{ Western China } & Tibetans & \multirow{2}{*}{$\begin{array}{l}\text { rs } 2296651 \\
\text { rs } 4646287 \\
\text { rs7154439 }\end{array}$} & 432 & 439 & $53.9 / 67.4$ & $40[33,55] / 39[30,49]^{\mathrm{C}}$ & \multirow{2}{*}{ TD PCR } & 6 \\
\hline & & Uygurs & & 234 & 197 & $59.8 / 61.9$ & $48[39,58] / 29[23,42]^{\mathrm{C}}$ & & \\
\hline Wu, W. (2017) (11) & Southern China & Han & $\begin{array}{l}\text { rs2296651 } \\
\text { rs7154439 }\end{array}$ & 595 & 586 & $65.0 / 73.7$ & $(45.53 \pm 11.95)\left((45.68 \pm 11.53)^{\mathrm{a}}\right.$ & iMLDR & 8 \\
\hline Zhang, Y.(2017) (13) & Southeastern China & Han & $\begin{array}{c}\text { rs } 2296651 \\
\text { rs } 4646287 \\
\text { rs } 7154439\end{array}$ & 2116 & 2535 & $74.1 / 73.1$ & $(41.3 \pm 13.8)(41.2 \pm 12.1)^{\mathrm{a}}$ & $\begin{array}{l}\text { Mass } \\
\text { Array }\end{array}$ & 7 \\
\hline Su, M. (2017) (12) & Southern China & Han & $\begin{array}{l}\text { rs2296651 } \\
\text { rs4646287 }\end{array}$ & 199 & 305 & $47.2 / 70.8$ & $(45.4 \pm 11.3)\left((42.2 \pm 13.9)^{\mathrm{a}}\right.$ & iMLDR & 6 \\
\hline Chuaypen, N. (2019) (15) & Thailand & Thai & $\begin{array}{l}\text { rs2296651 } \\
\text { rs4646287 }\end{array}$ & 206 & 610 & $66.5 / 69.7$ & $(51.3 \pm 5.9) /(52.6 \pm 12.2)^{\mathrm{a}}$ & TaqMan & 7 \\
\hline
\end{tabular}

Abbreviations: iMLDR, improved multiplex ligation detection reaction; NA, not available; NOS, Newcastle-Ottawa scale; SNP, single nucleotide polymorphism; TD PCR, touchdown polymerase chain reaction.

${ }^{\mathrm{a}}$ Values are expressed as mean $\pm \mathrm{SD}$.

b Values are expressed as mean (range).

${ }^{\text {Values are expressed as median (interquartile range) }}$

The current study also indicated that the frequency of rs4646287 genotype AA in the persistent infection group was slightly higher than that of the control group, but the difference was not statistically significant, which may indicate that rs 4646287 gene polymorphism is not associated with susceptibility to HBV. In terms of disease progression, 


\begin{tabular}{|c|c|c|c|c|c|c|c|c|}
\hline SNP & Number of Studies & Genetic Model & & PValue & OR $(95 \% \mathrm{CI})$ & $\mathbf{I}^{2}(\%)$ & Q-test & Egger's $P$ \\
\hline \multirow{3}{*}{ rs2296651 } & \multirow{3}{*}{7} & Dominant & GG vs. GA+AA & $0.010^{\mathrm{a}}$ & $0.73(0.58,0.93)$ & 51.1 & 0.046 & 0.277 \\
\hline & & Codominant & GG vs. GA & $0.008^{\mathrm{a}}$ & $0.74(0.59,0.93)$ & 45.2 & 0.078 & 0.216 \\
\hline & & Allele & G vs. A allele & $0.014^{\mathrm{a}}$ & $0.74(0.58,0.94)$ & 56.0 & 0.026 & 0.332 \\
\hline \multirow{6}{*}{ rs4646287 } & \multirow{6}{*}{5} & Dominant & GG vs. GA+AA & 0.150 & $1.10(0.97,1.24)$ & 0.0 & 0.420 & 0.849 \\
\hline & & Recessive & GG+GA vs. AA & 0.332 & $1.26(0.79,2.02)$ & 14.1 & 0.324 & 0.862 \\
\hline & & Codominant1 & GG vs. AA & 0.304 & $1.28(0.80,2.05)$ & 14.1 & 0.325 & 0.874 \\
\hline & & Codominant2 & GAvs. AA & 0.530 & $1.17(0.72,1.89)$ & 17.0 & 0.304 & 0.755 \\
\hline & & Codominant3 & GG vs. GA & 0.206 & $1.09(0.96,1.24)$ & 0.0 & 0.810 & 0.239 \\
\hline & & Allele & Gvs. A allele & 0.116 & $1.10(0.98,1.23)$ & 0.0 & 0.765 & 0.719 \\
\hline \multirow{6}{*}{ rs7154439 } & \multirow{6}{*}{5} & Dominant & GG vs. GA+AA & 0.323 & $1.04(0.96,1.14)$ & 0.0 & 0.940 & 0.795 \\
\hline & & Recessive & GG+GA vs. AA & 0.109 & $0.72(0.48,1.07)$ & 60.3 & 0.028 & 0.076 \\
\hline & & Codominant1 & GG vs. AA & 0.136 & $0.75(0.51,1.10)$ & 57.1 & 0.040 & 0.080 \\
\hline & & Codominant2 & GAvs. AA & 0.069 & $0.67(0.43,1.03)$ & 63.9 & 0.016 & 0.066 \\
\hline & & Codominant3 & GG vs. GA & 0.134 & $1.07(0.98,1.17)$ & 0.0 & 0.774 & 0.140 \\
\hline & & Allele & G vs. A allele & 0.739 & $1.01(0.94,1.09)$ & 0.0 & 0.718 & 0.293 \\
\hline
\end{tabular}

Abbreviation: SNP, single nucleotide polymorphism.

${ }^{\mathrm{a}} \mathrm{P}<0.05$.

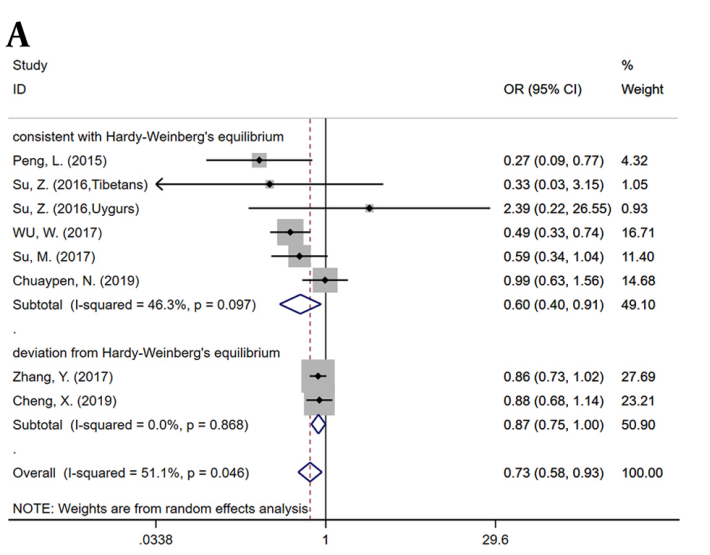

$\underset{\text { suco }}{\mathbf{C}}$

10

consistent with Hardy-Weinberg's equilibrium

Peng, L. (2015)

Su, Z. (2016, Tibetans)

Su, Z. (2016,Uygurs)

WU, W. (2017)

$\mathrm{Su}, \mathrm{M}$. (2017)

Chuaypen, N. (2019)

Subtotal (1-squared $=57.7 \%, p=0.037)$

deviation from Hardy-Weinberg's equilibrium

Zhang, Y. (2017)

Cheng, X. (2019)

Subtotal (l-squared $=0.0 \%, p=0.783$ )

Overall (I-squared $=56.0 \%, p=0.026$ )

NOTE: Weights are from random effects analysis
B

\begin{tabular}{|c|c|c|}
\hline $\begin{array}{l}\text { Study } \\
\text { ID }\end{array}$ & OR $(95 \% \mathrm{Cl})$ & $\begin{array}{l}\% \\
\text { Weight }\end{array}$ \\
\hline \multicolumn{3}{|l|}{ consistent with Hardy-Weinberg's equilibrium } \\
\hline Peng, L. (2015) & $0.28(0.10,0.83)$ & 3.86 \\
\hline \multicolumn{3}{|l|}{ Su, Z. (2016,Tibetans) $\longleftarrow$} \\
\hline Su, Z. (2016, Uygurs) & $2.39(0.22,26.55)$ & 0.83 \\
\hline WU, W. (2017) & $0.51(0.34,0.77)$ & 16.34 \\
\hline Su, M. (2017) & $0.59(0.34,1.04)$ & 10.82 \\
\hline Chuaypen, N. (2019) & $0.90(0.57,1.44)$ & 13.81 \\
\hline Subtotal $(1-$ squared $=27.9 \%, p=0.226)<$ & $0.61(0.43,0.85)$ & 46.60 \\
\hline \multicolumn{3}{|l|}{ deviation from Hardy-Weinberg's equilibrium } \\
\hline Zhang, Y. (2017) & $0.88(0.74,1.04)$ & 29.47 \\
\hline Cheng, X. (2019) & $0.88(0.68,1.14)$ & 23.93 \\
\hline Subtotal (l-squared $=0.0 \%, p=0.998)$ & $0.88(0.76,1.01)$ & 53.40 \\
\hline Overall (l-squared $=45.2 \%, p=0.078$ ) & $0.74(0.59,0.93)$ & 100.00 \\
\hline \multicolumn{3}{|l|}{ NOTE: Weights are from random effects analysis } \\
\hline .0338 & & \\
\hline
\end{tabular}

Figure 2. The forest plots for subgroup analysis of the association between rs2296651 polymorphism and spontaneous clearance of HBV. A, B and C, represent the dominant (GG vs. GA+AA), codominant (GG vs. GA) and allele genetic model (G vs. A), respectively. Odds ratios and respective $95 \%$ confidence intervals for the different studies included were shown. 

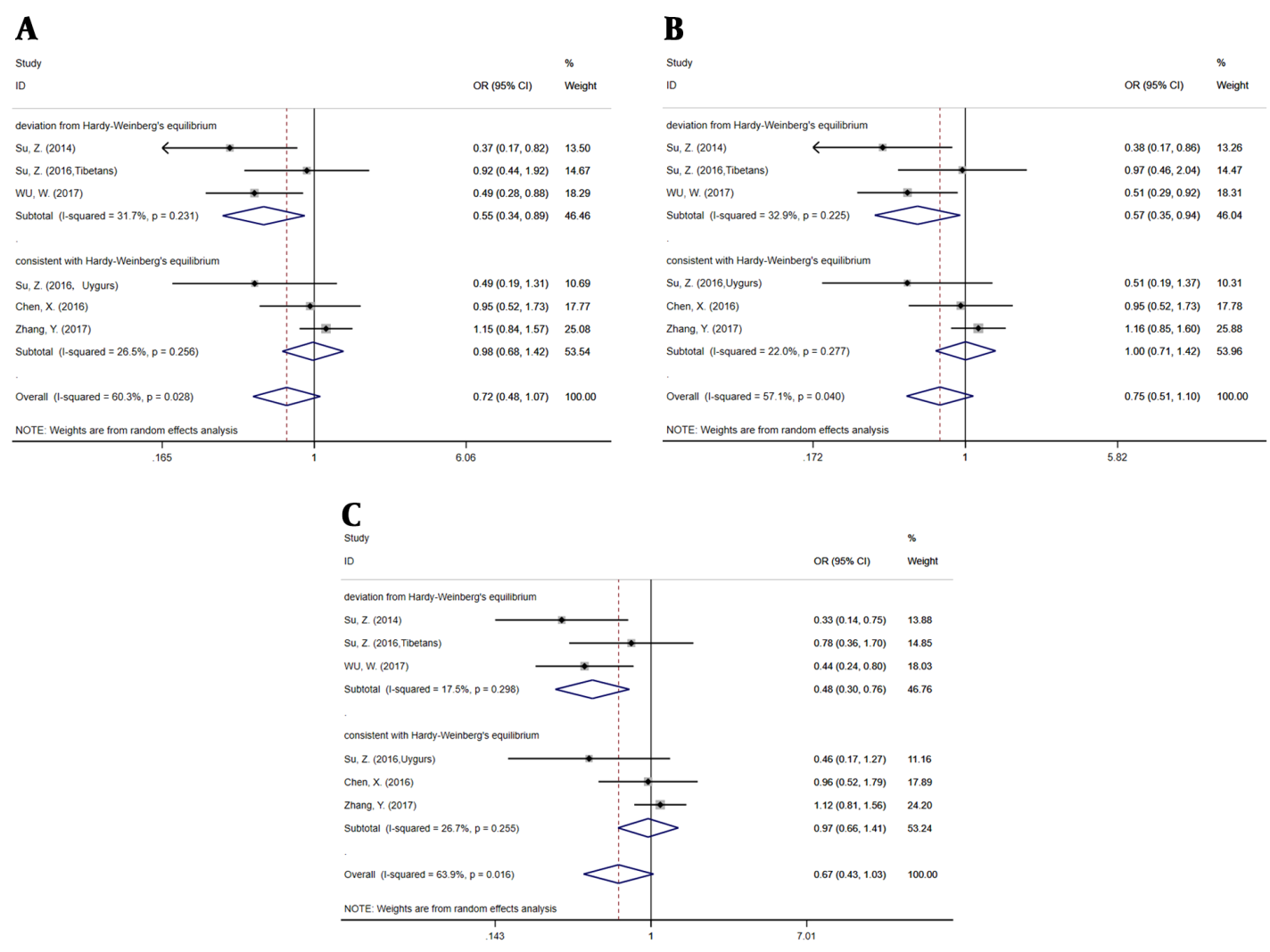

Figure 3. The forest plots for subgroup analysis of the association between rs7154439 polymorphism and spontaneous clearance of HBV. A, B and C, represent the recessive (GG+GA vs. AA), codominant1 (GG vs. AA) and codominant2 (GA vs. AA), respectively. Odds ratios and respective $95 \%$ confidence intervals for the different studies included were shown.

study by Su et al. (9) showed that rs4646287 gene polymorphism was associated with HBV-related cirrhosis and liver cancer.

As for SLC10A1 rs7154439, the current study results for all six models indicated no association between the polymorphism and HBV susceptibility. Furthermore, the combination of recessive, codominant model 1 and 2 in subgroup analysis showed the same results after limiting the study which satisfied HWE, and there was no significant heterogeneity among the studies. Although the deviation from HWE can partly explain the heterogeneity in the overall analysis, sensitivity analysis still suggested that the results were not robust. Given the limited number of studies available for the present meta-analysis, further studies are needed to assess the role of rs7154439 variation in the spontaneous clearance of HBV.

To the best of authors' knowledge, the current study is the first meta-analysis on the association of NTCP gene polymorphisms with HBV susceptibility. The control population in the current study was spontaneous clearance of $\mathrm{HBV}$ with a clear history of HBV virus infection. Yet, the meta-analysis also has the following limitations. First, local databases in other regions were not searched, which may lead to the inclusion of studies mostly focused on Asian populations (especially Chinese). Thus, subgroup analysis cannot be carried out to explore the association between NTCP gene polymorphisms and persistent HBV infection in different ethnical populations. Second, the lack of frequency data combined with genotypes and other environmental factors limited the study ability to further assess gene-environment interactions such as smoking and drinking.

In conclusion, the present meta-analysis results suggested that SLC10A1 rs2296651 polymorphism may play a significant role against persistent HBV infection in Asian populations, while the variation of NTCP gene rs4646287 was not associated with persistent HBV susceptibility. As for rs7154439, more researches are needed to confirm the current study results indicating no association between rs7154439 variant and spontaneous clearance. Further studies investigating the NTCP gene variant in other ethnic populations are also essential. 
A

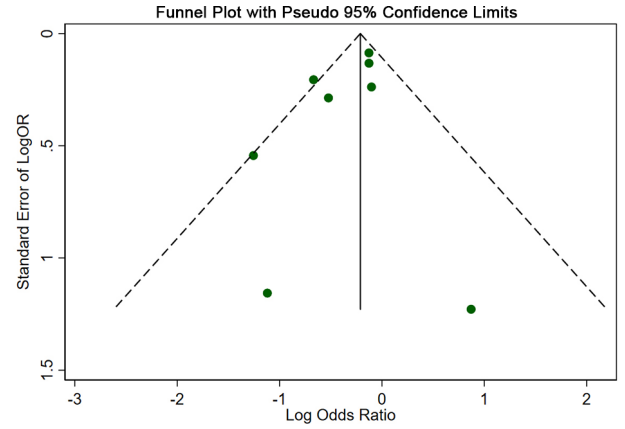

B

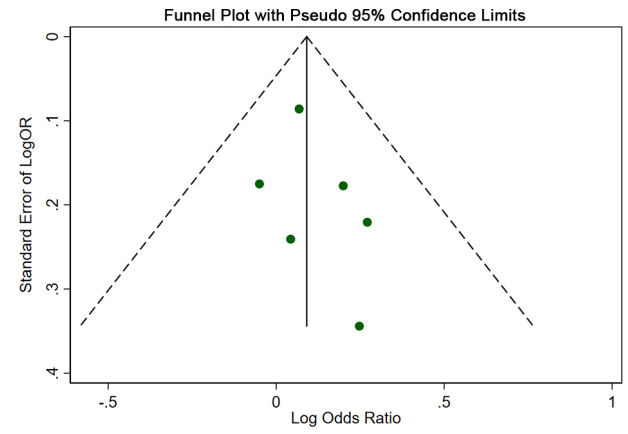

C

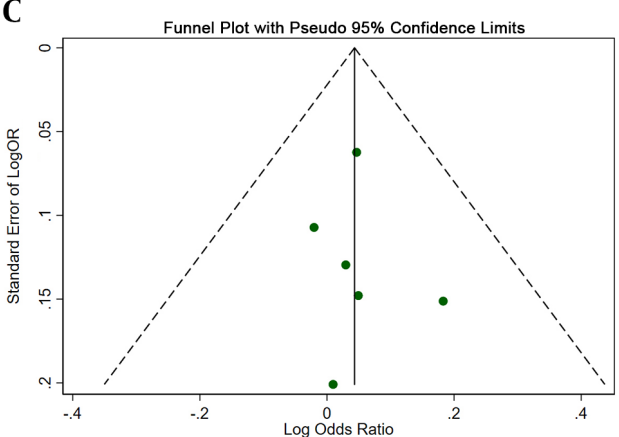

Figure 4. The funnel plots of the association between NTCP gene polymorphisms and spontaneous clearance of HBV under the dominant genetic model. A, B and C, represent rs2296651, rs4646287 and rs7154439, respectively.

\section{Supplementary Material}

Supplementary material(s) is available here [To read supplementary materials, please refer to the journal website and open PDF/HTML].

\section{Footnotes}

Authors' Contribution: Study concept and design: Peipei Hu, Dingmei Zhang; literature search, data extraction, and statistical analysis: Peipei Hu, Jundi Liu; interpretation of data and drafting of the manuscript: Peipei Hu; critical revision of the manuscript for important intellectual content: Dingmei Zhang.

Conflict of Interests: We declare that we have no conflict of interest.

Funding/Support: This work was supported by the National Science and Technology Major Project of the Ministry of Science and Technology of China (2018ZX10715004) and the Guangdong Provincial Science and Technology Program (2017A070713013). The funders had no role in study design, data collection and analysis, decision to publish, or preparation of the manuscript.

\section{References}

1. Schweitzer A, Horn J, Mikolajczyk RT, Krause G, Ott JJ. Estimations of worldwide prevalence of chronic hepatitis B virus infection: A systematic review of data published between 1965 and 2013. Lancet. 2015;386(10003):1546-55. doi: 10.1016/S0140-6736(15)61412-X. [PubMed: 26231459].

2. Tong MJ, Weiner JM, Ashcavai MW, Redeker AG, Comparini S, Vyas GN. A comparative study of hepatitis B viral markers in the family members of Asian and non-Asian patients with hepatitis B surface antigenpositive hepatocellular carcinoma and with chronic hepatitis B infection. J Infect Dis. 1979;140(4):506-12. doi: 10.1093/infdis/140.4.506. [PubMed: 229173].

3. Lin TM, Chen CJ, Wu MM, Yang CS, Chen JS, Lin CC, et al. Hepatitis B virus markers in Chinese twins. Anticancer Res. 1989;9(3):737-41. [PubMed: 2764519].

4. Stieger B. The role of the sodium-taurocholate cotransporting polypeptide (NTCP) and of the bile salt export pump (BSEP) in physiology and pathophysiology of bile formation. Handb Exp Pharmacol. 2011;(201):205-59. doi: 10.1007/978-3-642-14541-4_5. [PubMed: 21103971].

5. Yan H, Zhong G, Xu G, He W, Jing Z, Gao Z, et al. Sodium taurocholate cotransporting polypeptide is a functional receptor for human hepatitis B and D virus. Elife. 2012;1. e00049. doi: 10.7554/eLife.00049. [PubMed: 23150796]. [PubMed Central: PMC3485615].

6. Yan H, Peng B, Liu Y, Xu G, He W, Ren B, et al. Viral entry of hepatitis $B$ and $D$ viruses and bile salts transportation share common molecular determinants on sodium taurocholate cotransporting polypeptide. J Virol. 2014;88(6):3273-84. doi: 10.1128/JVI.03478-13. [PubMed: 24390325]. [PubMed Central: PMC3957944]. 
7. Su Z, Li Y, Liao Y, Cai B, Chen J, Zhang J, et al. Polymorphisms in sodium taurocholate cotransporting polypeptide are not associated with hepatitis B virus clearance in Chinese Tibetans and Uygurs. Infect Genet Evol. 2016;41:128-34. doi: 10.1016/j.meegid.2016.03.039. [PubMed: 27051045].

8. Peng L, Zhao Q, Li Q, Li M, Li C, Xu T, et al. The p.Ser267Phe variant in SLC10A1 is associated with resistance to chronic hepatitis B. Hepatology. 2015;61(4):1251-60. doi: 10.1002/hep.27608. [PubMed: 25418280].

9. Su Z, Li Y, Liao Y, Cai B, Chen J, Zhang J, et al. Association of the gene polymorphisms in sodium taurocholate cotransporting polypeptide with the outcomes of hepatitis B infection in Chinese Han population. Infect Genet Evol.2014;27:77-82. doi: 10.1016/j.meegid.2014.07.001. [PubMed: 25010264].

10. Chen X, Wang Y, Chen X, Cheng K, Li J, Lou J, et al. Genetic variants in the regulatory region of SLC10A1 are not associated with the risk of hepatitis B virus infection and clearance. Infect Genet Evol. 2016;44:495-500. doi: 10.1016/j.meegid.2016.07.043. [PubMed: 27491457].

11. Wu W. [Exploring the key locis affecting hepatitis B infection in Chinese han population at NTCP and CYP7A1 genes and confirmed its biological function] [dissertation]. China: Fujian Medical University; 2017. Chinese.

12. Su M, Chen H, Zeng Y, Chen T, Chen J, Jiang L, et al. Association of genetic variation of CIITA and NTCP with chronic hepatitis B Virus infection in han Chinese populations. Hepat Mon. 2017;17(6). doi: 10.5812/hepatmon.33646.

13. Zhang Y, Li Y, Wu M, Cao P, Liu X, Ren Q, et al. Comprehensive assessment showed no associations of variants at the SLC10A1 locus with susceptibility to persistent HBV infection among Southern Chinese. Sci Rep. 2017;7:46490. doi: 10.1038/srep46490. [PubMed: 28429786]. [PubMed Central: PMC5399367].

14. Cheng X, Wang Y, Tian J, Zhou L, Chen X, Guo H, et al. SLC10A1 S267F variant influences susceptibility to HBV infection and reduces cholesterol level by impairing bile acid uptake. J Viral Hepat. 2019;26(10):1178-85. doi: 10.1111/jvh.13157. [PubMed: 31177598].

15. Chuaypen N, Tuyapala N, Pinjaroen N, Payungporn S, Tangkijvanich P. Association of NTCP polymorphisms with clinical outcome of hepatitis B infection in Thai individuals. BMC Med Genet. 2019;20(1):87. doi: 10.1186/s12881-019-0823-x. [PubMed: 31117968]. [PubMed Central: PMC6532194].

16. Ezzikouri S, Chihab H, Elhabazi A, Wakrim L, Benjelloun S. Lack of Ser267Phe variant of sodium taurocholate cotransporting polypeptide among Moroccans regardless of hepatitis B virus infection status. BMC Infect Dis. 2017;17(1):99. doi: 10.1186/s12879-017-2214-2. [PubMed: 28125961]. [PubMed Central: PMC5270288]. 